\title{
Essential role of TNF family molecule LIGHT as a cytokine in the pathogenesis of hepatitis
}

Sudarshan Anand, ${ }^{1}$ Pu Wang, ${ }^{2}$ Kiyoshi Yoshimura, ${ }^{3}$ In-Hak Choi, ${ }^{4}$ Anja Hilliard, ${ }^{2}$ Youhai H. Chen, ${ }^{2}$ Chyung-Ru Wang, ${ }^{5}$ Richard Schulick, ${ }^{6}$ Andrew S. Flies, ${ }^{7}$ Dallas B. Flies, ${ }^{1}$ Gefeng Zhu, ${ }^{7}$ Yanhui Xu, ${ }^{8}$ Drew M. Pardoll, ${ }^{3}$ Lieping Chen, ${ }^{1,3,7,9}$ and Koji Tamada7

${ }^{1}$ Immunology Graduate Program, Johns Hopkins University School of Medicine, Baltimore, Maryland, USA. ${ }^{2}$ Department of Pathology and Laboratory Medicine, University of Pennsylvania School of Medicine, Philadelphia, Pennsylvania, USA. ${ }^{3}$ Department of Oncology, Johns Hopkins University School of Medicine, Baltimore, Maryland, USA. ${ }^{4}$ Department of Microbiology, Inje University College of Medicine, Pusan, Republic of Korea.

${ }^{5}$ Committee on Immunology and Department of Pathology, University of Chicago, Chicago, Illinois, USA. ${ }^{6}$ Department of Surgery and ${ }^{7}$ Department of Dermatology, Johns Hopkins University School of Medicine, Baltimore, Maryland, USA. ${ }^{8}$ Biochemistry Graduate Program, Mayo Clinic College of Medicine, Rochester, Minnesota, USA. Institute for Cell Engineering, Johns Hopkins University School of Medicine, Baltimore, Maryland, USA.

\begin{abstract}
LIGHT is an important costimulatory molecule for $T$ cell immunity. Recent studies have further implicated its role in innate immunity and inflammatory diseases, but its cellular and molecular mechanisms remain elusive. We report here that LIGHT is upregulated and functions as a proinflammatory cytokine in 2 independent experimental hepatitis models, induced by concanavalin A and Listeria monocytogenes. Molecular mutagenesis studies suggest that soluble LIGHT protein produced by cleavage from the cell membrane plays an important role in this effect through the interaction with the lymphotoxin- $\beta$ receptor (LT $\beta R$ ) but not herpes virus entry mediator. NK1.1 ${ }^{+} \mathrm{T}$ cells contribute to the production, but not the cleavage or effector functions, of soluble LIGHT. Importantly, treatment with a mAb that specifically interferes with the LIGHT-LT $\beta$ R interaction protects mice from lethal hepatitis. Our studies thus identify a what we believe to be a novel function of soluble LIGHT in vivo and offer a potential target for therapeutic interventions in hepatic inflammatory diseases.
\end{abstract}

\section{Introduction}

Molecules belonging to the TNF superfamily play an integral role in the regulation of innate and adaptive immunity, as well as contributing to inflammatory responses through their effects on nonhematopoietic cells (1). LIGHT (homologous to lymphotoxin, exhibits inducible expression, and competes with HSV glycoprotein $\mathrm{D}$ for herpes virus entry mediator, a receptor expressed by $\mathrm{T}$ lymphocytes) is a recently identified type II transmembrane glycoprotein of the TNF ligand superfamily (2). LIGHT is expressed on immature DCs and activated T cells $(2,3)$ and binds to 3 distinct receptors, herpes virus entry mediator (HVEM), lymphotoxin- $\beta$ receptor $(\mathrm{LT} \beta \mathrm{R})$, and decoy receptor $3 / \mathrm{TR} 6(2,4)$. Upon binding to HVEM, LIGHT costimulates $\mathrm{T}$ cells and accelerates proliferation and cytokine production $(3,5)$. Gene targeting of LIGHT results in impaired $\mathrm{T}$ cell immunity and a compromised allograft rejection (6-8). Thus, there is ample evidence indicating a crucial role of LIGHT in the adaptive immune system.

In contrast, the functional role of LIGHT in innate and inflammatory responses remains less explored. Although it has been shown in vitro that LIGHT induces apoptosis in several tumor cell lines, there are conflicting reports in regard to the specific receptor mediating this effect $(9,10)$. Recent studies have suggested a potential role of LIGHT in inflammatory diseases including atherosclerosis (11), arthritis (12), IgA nephropathy (13), and inflammatory bowel disease (14). Despite these lines of investigation, the mechanism by which LIGHT mediates inflammatory responses remains

Nonstandard abbreviations used: ALT, alanine aminotransferase; B6, C57BL/6J; BTLA, B and T lymphocyte attenuator; ConA, concanavalin A; HVEM, herpes virus entry mediator; LIGHT, homologous to lymphotoxin, exhibits inducible expression, and competes with HSV glycoprotein D for herpes virus entry mediator, a receptor expressed by $\mathrm{T}$ lymphocytes; LT $\beta \mathrm{R}$, lymphotoxin- $\beta$ receptor.

Conflict of interest: The authors have declared that no conflict of interest exists. Citation for this article: J. Clin. Invest. 116:1045-1051 (2006). doi:10.1172/JCI27083. elusive. For instance, we do not know which receptor(s) of LIGHT is responsible for these effects. In addition, although the soluble form of LIGHT has been detected in vitro $(15,16)$, its presence and functions in the inflammatory diseases have yet to be explored in vivo.

Liver inflammation is mediated by immune responses to hepatocytes following liver-tropic pathogen infections or by pathogenic autoreactivity. Exploration of molecular and cellular mechanisms underlying liver inflammation is essential to treat acute hepatitis and to prevent liver cirrhosis and hepatocellular carcinoma following chronic hepatitis. A number of regulators have been implicated in both human and experimental hepatitis, including TNF superfamily molecules such as TNF- $\alpha$ and FasL (17). In addition, potential involvement of LIGHT and LT $\beta$ R in liver inflammation has been suggested by several observations. First, mice overexpressing LIGHT show immune cell infiltrations in the periportal areas of the liver $(18,19)$. Second, enhanced expression of lymphotoxin- $\beta$ is detected in the liver of patients with hepatitis C virus infection (20). Interestingly, a direct molecular interaction between the cytoplasmic domain of LT $\beta R$ and hepatitis $C$ virus core protein has been observed (21-23). Finally, LIGHT-LT $\beta$ R interaction is implicated in the apoptotic cell death of hepatoma cells (24) as well as in the regeneration of hepatocytes (25). These studies together suggest a potential regulatory function of LIGHT in liver inflammation.

In this study, we explore the expression and pathogenic role of LIGHT in 2 distinct mouse hepatitis models. We investigate the molecular mechanism of LIGHT-mediated liver inflammation and offer a novel therapeutic strategy based on this mechanism to ameliorate inflammation in the liver.

\section{Results}

Increased expression and a pathogenicrole for LIGHTinexperimental hepatitis. In order to study a potential role for LIGHT in liver inflammation, we took advantage of a mouse hepatitis model induced by 

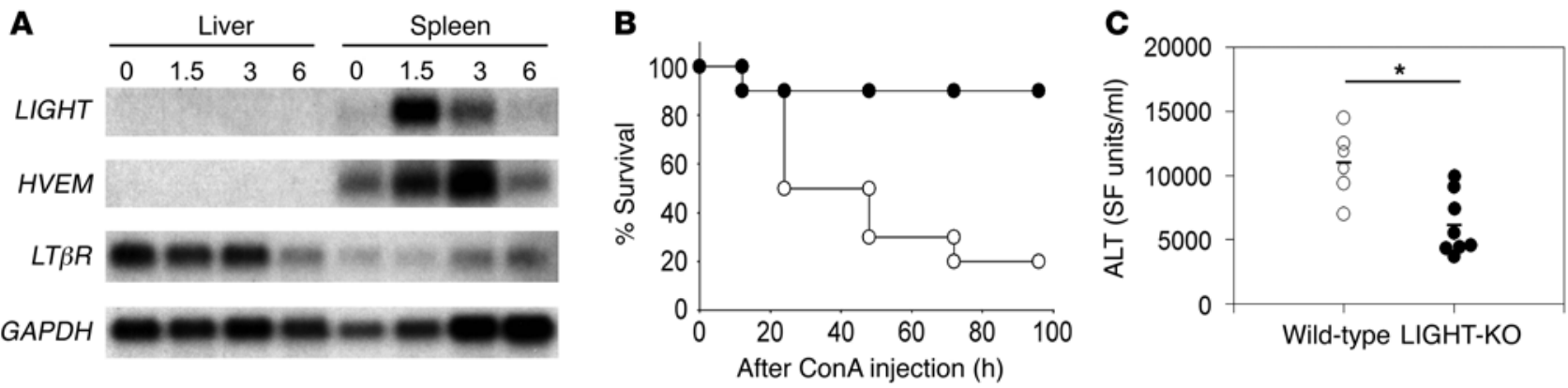

\section{Figure 1}

Pathogenic role of upregulated LIGHT in hepatitis. (A) BALB/c mice were injected i.v. with $25 \mathrm{mg} / \mathrm{kg}$ ConA. At the indicated time points, the mice were sacrificed, and total RNA was extracted from liver and spleen. LIGHT, HVEM, LTRR, and GAPDH expression was examined by Northern blot analysis. (B and C) Wild-type (open circles) and LIGHT-deficient (filled circles) mice were injected i.v. with $30 \mathrm{mg} / \mathrm{kg}$ ConA. The survival (B) and serum ALT levels (C) of recipient mice were monitored. Sera were collected 18 hours after ConA injection, and the ALT levels were measured as described in Methods. SF, Sigma-Frankel. ${ }^{*} P=0.003$.

concanavalin A (ConA) injection (26). First, we investigated the expression of LIGHT and its receptors in this model. Northern blot analysis showed that 1.5-3 hours after ConA treatment, LIGHT and HVEM mRNA expression was upregulated predominantly in the spleen, while $L T \beta R$ was constitutively expressed in the liver, irrespective of ConA treatment (Figure 1A). This finding indicated an enhanced expression of LIGHT in the early phase of experimental acute hepatitis, leading us to further investigate the pathogenic role of LIGHT in the liver.

Using LIGHT-deficient mice (7), we then examined whether the increase of LIGHT expression was responsible for the pathogenesis of ConAinduced hepatitis. Four days after injection of a lethal dose of ConA, 80\% of control C57BL/6J (B6) mice died of acute hepatitis (Figure 1B), which was characterized by a massive infiltration of inflammatory cells in the liver (data not shown) and an elevated serum alanine aminotransferase (ALT) (Figure 1C). In contrast, more than $90 \%$ of LIGHT-deficient mice survived indefinitely with significantly lower levels of ALT. These results clearly indicate that LIGHT expression is a crucial event for the initiation and/or progression of ConA-induced hepatitis.

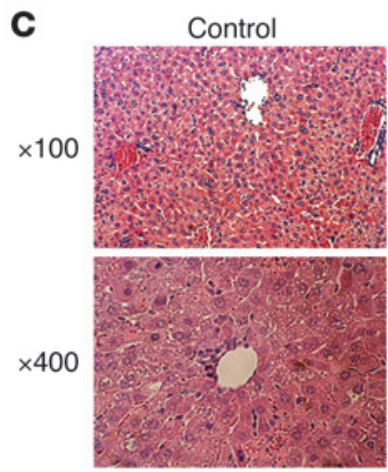

Figure 2 is shown as mean $\pm \mathrm{SD}$.
Proinflammatory functions of soluble LIGHT as a cytokine. In order to explore the mechanisms underlying LIGHT-mediated liver inflammation, we raised 3 crucial questions to be addressed. The first is whether the soluble form of LIGHT contributes to the liver pathogenesis, since LIGHT is cleaved from the cell membrane by MMP along with inflammatory stimuli $(15,16)$. The second question is whether HVEM or LT $\beta$ R is responsible for
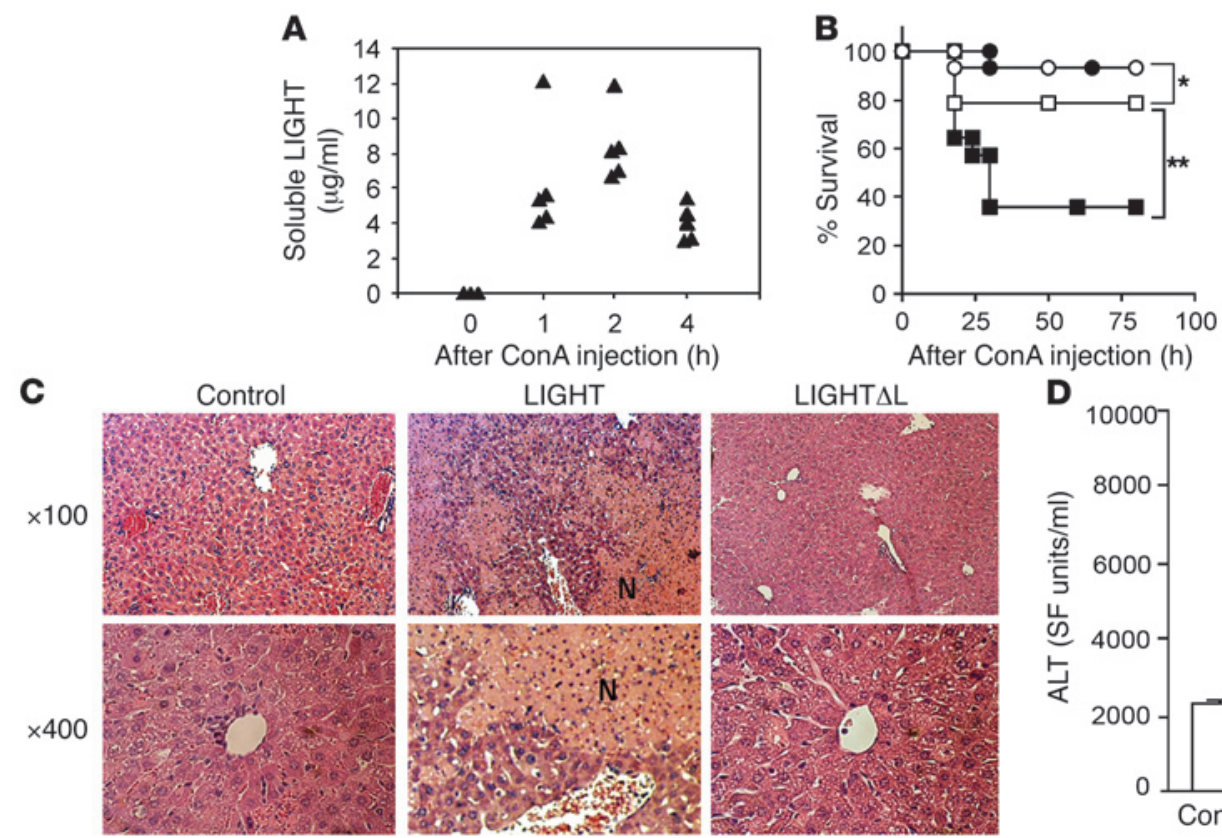

Essential role of the soluble form of LIGHT in liver inflammation. (A) B6 mice were injected i.v. with $30 \mathrm{mg} / \mathrm{kg}$ of ConA. At the indicated time points, serum was collected from the recipient mice and measured for soluble LIGHT concentration by LIGHT-specific ELISA. (B and C) B6 mice were injected with a sublethal dose of ConA $(12.5 \mathrm{mg} / \mathrm{kg})$ alone (filled circles, $n=13$ ) or together with $20 \mu \mathrm{g}$ plasmids encoding control pcDNA3.1 (open circles, $n=13$ ), wild-type LIGHT (filled squares, $n=11$ ), or LIGHT $\Delta$ L (open squares, $n=10$ ) by hydrodynamic injection technique. Survival of mice (B) and liver sections stained with H\&E 18 hours after injection (C) were examined. N, necrotic area. ${ }^{*} P=0.3,{ }^{* \star} P=0.033$ between the groups by log-rank test. (D) BALB/c mice were injected i.p. with $50 \mu \mathrm{g}$ of soluble LIGHT-flag fusion protein or control protein. One hour later, the mice were injected i.v. with $25 \mathrm{mg} / \mathrm{kg}$ ConA, and serum ALT levels were measured 6 hours later. One representative result from 3 independent experiments 
A

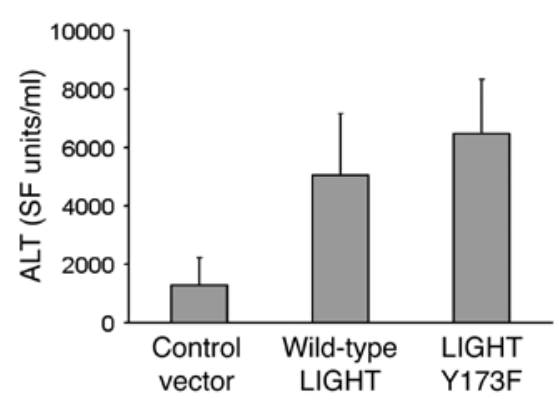

B

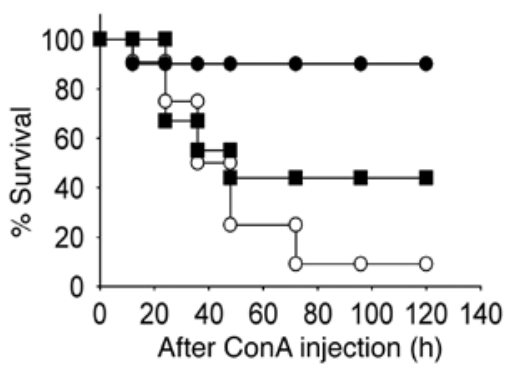

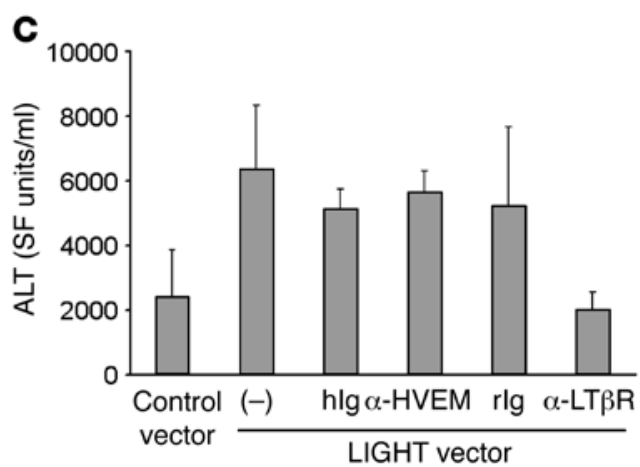

Figure 3

LT $\beta R$ is necessary and sufficient for LIGHT-mediated hepatitis. (A) B6 mice were injected with either empty vector or plasmid DNA encoding wild-type LIGHT or Y173F mutant by hydrodynamic method in combination with a sublethal dose of ConA (12.5 mg/kg). ALT levels were measured 18 hours after injection. One representative result from 2 independent experiments is shown as mean \pm SD of 5 mice per group. (B) B6 mice were injected i.v. with $30 \mathrm{mg} / \mathrm{kg}$ of ConA together with $100 \mu \mathrm{g}$ of control rat Ig (open circles, $n=12$ ), anti-LT $\beta R$ (filled circles, $n=10$ ), or anti-HVEM mAb (filled squares, $n=9$ ). The survival was monitored thereafter. (C) B6 mice were injected with either empty vector or plasmid DNA encoding LIGHT by hydrodynamic method in combination with a sublethal dose of ConA $(12.5 \mathrm{mg} / \mathrm{kg})$. The mice were treated i.p. with $100 \mu \mathrm{g}$ of the indicated Abs 2 hours before the plasmid injections. After 18 hours, serum ALT levels were measured. One representative result from 2 independent experiments is shown as mean \pm SD of 5 mice per group. hlg, control hamster lg; rlg, control rat Ig.

this effect as a functional receptor. The third one is how and which cells produce LIGHT that mediates liver inflammation.

To address the first question, we examined whether the soluble form of LIGHT is detectable in vivo upon ConA treatment. By a sandwich ELISA specific to mouse LIGHT, soluble LIGHT was detected in the mouse sera as soon as 1 hour after ConA treatment, followed by a rapid decline after 4 hours (Figure 2A), suggesting a potential role of soluble LIGHT in the pathogenesis of liver inflammation. To further ascertain this, we designed a LIGHT mutant resistant to the enzymatic cleavage. LIGHT $\Delta \mathrm{L}$, a deletion mutant lacking amino acids from Leu63 to Asp84, loses the capacity to produce soluble LIGHT both in vitro upon transfection into $293 \mathrm{~T}$ cells and in in vivo gene expression by hydrodynamic injection (27); rather, it preferentially resides on the cell membrane (Supplemental Figure 1; supplemental material available online with this article; doi:10.1172/JCI27083DS1). This mutant is not functionally compromised, as it can cause cell death in HT-29 cells in the presence of IFN- $\gamma$ at levels similar to those required of full-length LIGHT (Supplemental Figure 2). Together with sublethal ConA treatment, in vivo expression of wild-type LIGHT by hydrodynamic injection resulted in a high mortality of recipient mice (Figure $2 \mathrm{~B}$ ). In contrast, the mice expressing LIGHT $\Delta$ L survived as well as the mice receiving control vector plasmids. Consistent with these findings, pathological analyses indicated that hepatocytes were largely free from necrotic cell death after LIGHT $\Delta$ L expression, whereas there were massive necrotic foci of hepatocytes after wild-type LIGHT expression (Figure 2C). In addition, direct administration of recombinant soluble LIGHT protein significantly increased the serum ALT level at 6 hours (Figure 2D) and led to the death of all the mice by 24 hours (data not shown). Collectively, these findings suggest that soluble LIGHT production by the cleavage of the membrane-bound form is a crucial mechanism for hepatitis progression.

Indispensable role of $L T \beta R$, but not HVEM, in the proinflammatory effects of LIGHT. We next addressed whether HVEM or LT $\beta$ R plays a responsible role in liver inflammation as a functional receptor of soluble LIGHT. To this end, we generated another mutant of mouse LIGHT, Y173F, by a single-amino-acid substitution of Phe for Tyr173. LIGHT Y173F selectively loses the binding to HVEM but not LT $\beta$ R (Supplemental Figure 3), consistent with the analogous mutant of human LIGHT (9). Upon hydrodynamic injection into mice with a sublethal dose of ConA, LIGHT Y173F mediated hepatitis at a level comparable to that of wildtype LIGHT (Figure 3A), suggesting a dispensable role of HVEM in LIGHT-mediated hepatitis.

To further confirm this notion, we generated antagonistic mAbs to LT $\beta$ R or HVEM. Our anti-LT $\beta$ R Ab selectively blocks LIGHTLT $\beta \mathrm{R}$ interaction but not LT $\beta$-LT $\beta$ R interaction, while anti-HVEM
A

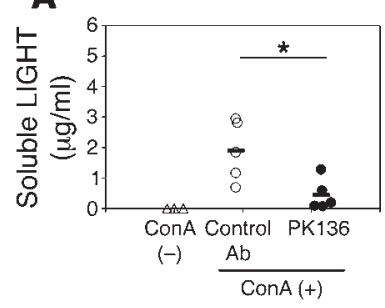

Figure 4

Essential role of NKT cells in the production of soluble LIGHT in ConA-induced hepatitis. (A and B) B6 mice were treated i.p. with $500 \mu \mathrm{g}$ of either control mouse lgG or anti-NK1.1 mAb (PK136) on days 0 and 3 (A). Similarly, B6 mice were treated i.p. with $30 \mu \mathrm{g}$ of control rabbit IgG or anti-asialo GM1 (ASGM1) on days 0 and 3 (B). On day 4 , each group was injected i.v. with $30 \mathrm{mg} / \mathrm{kg}$ of ConA, and mouse sera were collected 1 hour later. The amounts of soluble LIGHT were measured by ELISA. ${ }^{*} P=0.02$. (C) CD1d-deficient mice were injected with $20 \mu \mathrm{g}$ of control vector or LIGHT-encoding plasmid by hydrodynamic injection in combination with a sublethal dose of ConA $(12.5 \mathrm{mg} / \mathrm{kg})$. Mouse sera were collected 18 hours later, and ALT levels were measured. ${ }^{*} P=0.002$. 
A

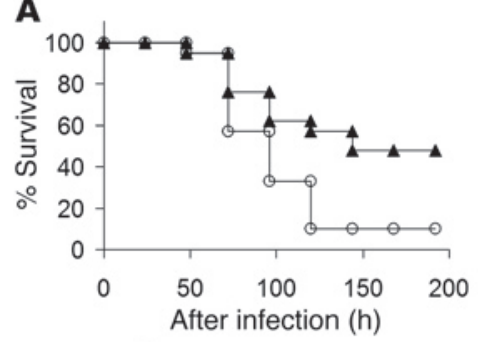

C Control IgG

$\times 100$

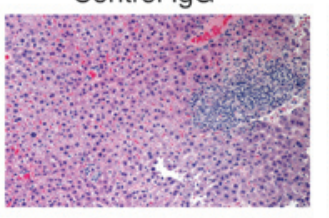

$\times 400$

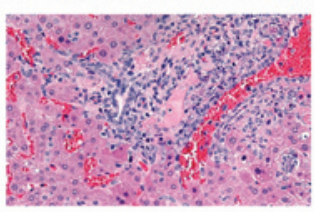

B

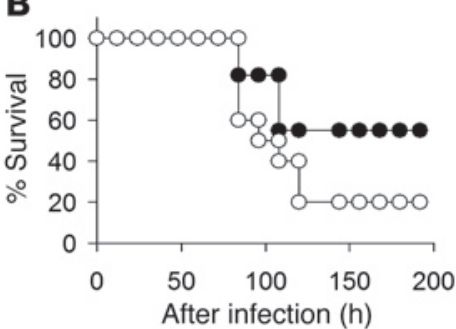

Anti-LT $\beta R$ mAb

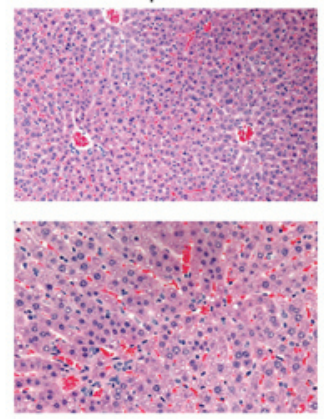

\section{Figure 5}

Blockade of LIGHT-LT $\beta R$ interaction as a treatment for L. monocytogenesinduced hepatitis. (A) B6 mice (open circles, $n=21$ ) and LIGHT-deficient mice (filled triangles, $n=21)$ were injected i.p. with $L$. monocytogenes $\left(2 \times \mathrm{LD}_{50}\right.$ per mouse), and their survival was monitored. $P=0.007$ between the groups. (B) B6 mice were injected i.v. with $100 \mu \mathrm{g}$ of control IgG (open circles, $n=10$ ) or antiLT $\beta R$ mAb (filled circles, $n=11$ ). At the same time, the mice were infected with L. monocytogenes $\left(2 \times \mathrm{LD}_{50}\right.$ per mouse) by i.p. injection. Survival of mice was monitored thereafter. $P=0.03$ between the groups. (C) As in B, B6 mice were infected with $L$. monocytogenes and treated with either anti-LT $\beta R$ mAb or control IgG. Three days after infection, liver sections were prepared and stained with H\&E.

$\mathrm{mAb}$ interferes with LIGHT-HVEM interaction but not interaction between B and T lymphocyte attenuator (BTLA) and HVEM (Supplemental Figure 4). Injection of anti-LTRR mAb profoundly decreased the mortality of mice with hepatitis induced by a lethal dose of ConA, whereas the anti-HVEM Ab showed a marginal effect on the survival (Figure 3B). Similarly, in the hepatitis model induced by hydrodynamic injection of LIGHT with sublethal ConA treatment, blockade of LIGHT-LT $\beta$ R, but not LIGHT-HVEM, resulted in a significant decrease of the serum ALT level (Figure 3C). Taken together, these findings indicate that LIGHT-LT $\beta$ R interaction is necessary and sufficient for LIGHT-mediated liver inflammation.

Essential role of NK1.1 $1^{+}$cells in the production of soluble LIGHT in ConA-induced hepatitis. Since a crucial role of NK1. $1^{+} \mathrm{T}$ cells (NKT cells) in the pathogenesis of ConA-induced hepatitis has been described $(28,29)$, we next examined a potential role of NKT cells in the production of soluble LIGHT. Mice treated with anti-NK1.1 $\mathrm{mAb}$, which depletes NK and NKT cells in vivo $(30,31)$, expressed significantly lower amounts of soluble LIGHT than the control Abtreated mice in response to ConA injection (Figure 4A). In contrast, the mice treated with asialo GM1, which depletes NK but not NKT cells (29), produced as much soluble LIGHT as those treated with control Ab (Figure 4B), suggesting a crucial role of NKT cells in the production of soluble LIGHT. On the other hand, when LIGHT was exogenously expressed by hydrodynamic injection, CD1d-deficient mice lacking NKT cells (32) showed a significant increase of serum ALT (Figure 4C), indicating a dispensable role of NKT cells

in the effector phase of LIGHT-mediated hepatitis. In addition, given that full-length LIGHT was expressed in this experiment, NKT cells may not be required for the cleavage of membrane-bound LIGHT. In fact, soluble LIGHT was detected at normal levels in anti-NK1.1 $\mathrm{mAb}$-treated mice after hydrodynamic expression of full-length LIGHT (Supplemental Figure 5). Furthermore, exogenous expression of LIGHT mediates a certain level of hepatitis even in the absence of ConA or in mice deficient in the Rag gene (Supplemental Figure 6), suggesting that cleavage and effector functions of soluble LIGHT require neither other inflammatory stimuli nor adaptive immune cells. Taken together, our findings indicate that NKT cells function as a crucial cellular source of LIGHT for hepatitis, rather than a regulator of cleavage or effector cells downstream of LIGHT.

Therapeutic potential of the LIGHT-LTBR pathway in Listeria monocytogenes-induced hepatitis. Lastly, we evaluated the pathogenic role and therapeutic potential of LIGHT in an acute hepatitis model induced by Listeria monocytogenes. Intraperitoneal infection with high-dose L. monocytogenes $\left(2 \times \mathrm{LD}_{50}\right.$ per mouse $)$ causes acute hepatitis associated with an immune cell infiltration and hepatocyte necrosis, ultimately leading to death in about 5 days (33). When we challenged wild-type or LIGHTdeficient $\mathrm{B} 6$ mice with this dose of $L$. monocytogenes, we found that the survival of LIGHT-deficient mice was significantly longer than that of the control mice (Figure 5A). Similarly, selective blockade of LIGHTLT $\beta R$ interaction by our anti-LT $\beta R$ mAb significantly prolonged the survival of high-dose L. monocytogenesinfected mice compared with those treated with control $\mathrm{Ab}$ (Figure 5B). This effect was not due to an enhanced clearance of bacteria, since the titers of $L$. monocytogenes in liver and spleen were comparable between anti-LT $\beta \mathrm{R} m A b-$ and control Ab-treated mice (data not shown). In addition, we detected a low but significant amount of soluble LIGHT in the serum 24 hours after infection $(100 \pm 70 \mathrm{ng} / \mathrm{ml}, n=5)$. Pathological analyses revealed that anti-LT $\beta \mathrm{R} m A b$ treatment decreased the infiltration of inflammatory cells and the necrosis of hepatocytes, thus maintaining overall integrity of liver microstructure (Figure 5C). These findings indicate that LIGHT plays a pathological role in L. monocytogenes-induced hepatitis and that blockade of the LIGHT-LT $\beta$ R pathway has a therapeutic potential in hepatic inflammation mediated by liver-tropic pathogen infections.

\section{Discussion}

In this study, we delineated a novel function of LIGHT as a proinflammatory cytokine for hepatocyte damage. During the course of experimental hepatitis, activation of immune cells, predominantly NKT cells, leads to LIGHT production and subsequent cleavage as a cytokine. Soluble LIGHT circulates systemically and mediates hepatocyte death through the interaction with LT $\beta$ R. Selective blockade of LIGHT-LT $\beta$ R interaction ameliorates experimental hepatitis induced by ConA or L. monocytogenes, attesting to the important, nonredundant role of this mechanism in promoting liver inflammation.

Although a potential role of the interaction of LIGHT with HVEM and LT $\beta R$ in various inflammatory diseases has been suggested (11-14), the pathogenic effects and underlying mechanism 
of this role have not been fully explored so far. In this study, using LIGHT-deficient mice, we observed an essential role of LIGHT in initiating and/or promoting inflammatory responses, rather than its being merely a by-product of the inflammatory cascade. Since LIGHT-deficient mice display a phenotype of impaired $\mathrm{T}$ cell immunity (6-8), it is worth considering whether lymphocyte activation through LIGHT costimulatory effects contributes to the inflammatory responses. In this regard, our findings using anti-HVEM $\mathrm{mAb}$ as well as LIGHT Y173F mutant suggest that a costimulatory role through HVEM is not necessary for LIGHTmediated liver inflammation. This notion could also be supported by evidence that LIGHT-deficient mice exhibit an immune dysfunction predominantly in $\mathrm{CD}^{+} \mathrm{T}$ cells $(6-8)$, whereas $\mathrm{CD}^{+}$ $\mathrm{T}$ cells and NKT cells are the central players in ConA-induced hepatitis $(26,28)$. In a model of $L$. monocytogenes-induced hepatitis, we found that the difference in survival of LIGHT-deficient mice becomes evident between days 3 and 5 after infection, when adaptive immunity including pathogen-specific $\mathrm{CD}^{+} \mathrm{T}$ cell responses has not yet dominated (33). In addition, selective blockade of LIGHT-LT $\beta$ R with anti-LT $\beta$ R mAb ameliorated hepatitis as effectively as LIGHT deficiency did, suggesting a negligible role of the HVEM costimulatory pathway. Interestingly, a recent study reported that HVEM-deficient mice have enhanced responses to ConA with severe damage of the spleen, but not the liver, and elevated levels of serum cytokines (34). These phenotypes are likely due to a lack of inhibitory signal through BTLA, another binding partner of HVEM (35). In light of these findings, it can be postulated that LIGHT mediates inflammatory responses through LT $\beta$ R, but not HVEM, on nonlymphocyte populations whereas HVEM-BTLA interaction negatively regulates inflammation by attenuating lymphocyte activation.

With regard to the cellular target of LIGHT-LT $\beta$ R interaction, there are 3 possible components in the liver: hepatocytes, Kupffer cells, and liver stromal cells. Primary hepatocytes have been shown to constitutively express LT $\beta R$ (25), although its role in initiating death is controversial $(24,36)$. It is plausible that, like peripheral macrophages (37), Kupffer cells express LT $\beta R$ and produce proinflammatory cytokines such as TNF- $\alpha$ in response to LIGHT signal, leading to hepatocyte death. Finally, liver stromal cells might produce chemoattractive factors by LIGHTLT $\beta$ R signaling, thereby accelerating infiltration of proinflammatory cells in the liver, as various nonhematopoietic cells have been reported to express chemokines and adhesion molecules in response to LT $\beta$ R stimulation (38). These possibilities are now under investigation in our laboratory.

The intracellular region of LT $\beta R$ has been shown to have specific domains for binding to TRAF-2, -3 , and -5 , which are responsible for the delivery of death signal (39). LIGHT-LT $\beta$ R interaction mediates cell death of certain tumor cell lines in the presence of IFN- $\gamma$, in which p38-MAPK and poly(ADP-ribose) polymerase (PARP) pathways induce upregulation of proapoptotic molecules such as Bak and downregulation of antiapoptotic molecules like Bcl-2 (40). In addition, the association of proapoptotic molecules like Smac and IAP-1, along with TRAF-2 and TRAF-3, with the endogenous LIGHT-LT $\beta$ R complex has been reported (41). More specifically, in a hepatoma cell line, Hep3B, it has been postulated that LIGHT mediates cell death via activation of ROS and Ask-1 downstream of the TRAF-3 and TRAF-5 signals from the LT $\beta R$ (42). At present, we have not explored whether these mechanisms are responsible for the death of hepatocytes in our hepatitis model, but it is likely that these molecular events play a synergistic role in the hepatocyte cell death.

Although there is evidence for the presence of soluble LIGHT cleaved by MMP $(15,16)$, to our knowledge, no studies have characterized the biological functions of soluble LIGHT. Here, our study using LIGHT mutant indicates that soluble LIGHT plays an important role in the pathogenesis of liver inflammation. Given that hydrodynamic injection of plasmid leads to a predominant gene expression in hepatocytes (43), the inability of LIGHT $\Delta$ L to mediate hepatitis suggests that a widespread distribution of soluble LIGHT, rather than cell-to-cell contact, might be essential to trigger inflammation against tightly packed cells such as hepatocytes. In addition, we detected soluble LIGHT in the serum after ConA injection (Figure 2A) and found the ability of soluble LIGHT to mediate hepatitis (Figure 2D), supporting a crucial function of soluble LIGHT. Indeed, this notion is consistent with previous reports that certain MMP inhibitors protect mice from the lethal hepatitis induced by endotoxin (44) or hepatotoxin (45). In clinical situations, a recent study indicated the presence of soluble LIGHT in the bronchoalveolar lavage of scleroderma patients with active inflammation but not in those without active inflammation (46). We also found low but detectable levels of soluble LIGHT in about $30 \%$ of patients chronically infected with hepatitis B virus (K. Tamada et al., unpublished data). Taken together, these findings suggest that soluble LIGHT functions as an important accelerator for tissue inflammation as well as a potential clinical marker for inflammatory activation.

There is substantial evidence that NKT cells play an indispensable role in the pathogenesis of ConA-induced hepatitis $(28,29)$. It was also suggested that the infection of $L$. monocytogenes is associated with the activation of NKT cells $(47,48)$. Although the mechanism of liver damage mediated by NKT cells has not been entirely explored, their expression of FasL and IFN- $\gamma$ may cause hepatocyte death directly or indirectly through the activation of Kupffer cells to produce TNF- $\alpha$ (49). Osteopontin derived from NKT cells and TNF-related apoptosis-inducing ligand (TRAIL) were also shown to be significant contributors to liver inflammation $(50,51)$. Our study here indicated that NKT cells are essential for the production, but not the cleavage or effector functions, of soluble LIGHT induced by ConA injection. Interestingly, serum levels of IFN- $\gamma$ and TNF- $\alpha$, but not osteopontin, in response to ConA injection are significantly decreased in LIGHT-deficient mice (Supplemental Figure 7). Although we have not explored a potential involvement of these effects in LIGHT-mediated hepatitis, it is conceivable that inflammatory mediators downstream of LIGHT contribute to the pathogenesis directly or indirectly through the synergistic effects with LIGHT (24).

Hepatic inflammation and liver regeneration appear to be 2 sides of the same coin. TNF ligand superfamily molecules that mediate hepatic death and inflammation also accelerate liver regeneration, as shown by TNF- $\alpha$ and FasL $(52,53)$. A recent study by Anders et al. indicated that the interaction of LIGHT and LT $\beta$ with LT $\beta$ R also plays a crucial role in stimulating liver regeneration (25). This dual function of LIGHT on hepatocytes could explain seemingly contradictory findings that it can either protect hepatocytes from death (36) or cause apoptosis itself (24). Thus, LIGHT as well as TNF- $\alpha$ and FasL has integral functions in the regulation of liver homeostasis. It seems that these factors play nonredundant roles that cannot be compensated for by others, since manipulation of any of these factors significantly changes liver homeostasis. Our work thus identifies a critical role for soluble LIGHT-LT $\beta$ R 
interaction in the pathogenesis of liver inflammation. Selective regulation of this arm among interactions between LIGHT, LT $\beta$ R, HVEM, and BTLA would enhance our ability to intervene in the inflammatory diseases without affecting other important functions associated with these molecular pathways.

\section{Methods}

Mice, cell lines, and reagents. C57BL/6J (B6) and BALB/c mice were purchased from the National Cancer Institute (Frederick, Maryland, USA) and Jackson Laboratory, respectively. The mice deficient in CD1d or LIGHT (B6 background) were described previously $(7,32)$. Age-matched 5- to 8-week-old mice were used for all experiments. All the animal experiments described in this manuscript were approved by the Johns Hopkins Animal Care and Use Committee of the Johns Hopkins University School of Medicine. A20 and 293T cells were purchased from ATCC. LIGHT-flag, LT $\beta$ R-Ig, and HVEM-Ig fusion proteins were prepared as previously described (3, 5). Anti-mouse HVEM mAb (clone, LH1) and anti-mouse LT $\beta$ R mAb (clone, LLTB1) were generated in our laboratory as described before (54). PK136 hybridoma was purchased from ATCC, and $\mathrm{mAb}$ was purified as previously described (54). Anti-asialo GM1 was purchased from Cedarlane Laboratories Ltd. Mouse IgG, rat IgG, and human IgG were purchased from Sigma-Aldrich. Hamster IgG was purchased from Rockland Immunochemicals. All cDNA plasmids were purified by an EndoFree Maxi preparation kit (QIAGEN).

ConA-induced hepatitis. The mice were injected i.v. with either a lethal dose $(25-30 \mathrm{mg} / \mathrm{kg})$ or a sublethal dose $(12.5 \mathrm{mg} / \mathrm{kg})$ of ConA (Sigma-Aldrich) in PBS with or without hydrodynamic injection of plasmid DNA. Mouse survival and serum ALT level were monitored periodically. Serum ALT level was measured by a Transaminase kit (Sigma-Aldrich) according to the manufacturer's instructions. In some experiments, livers were harvested from the treated mice, fixed in formalin solution, and embedded with paraffin. The sections were stained with H\&E for histological study.

L. monocytogenes-induced hepatitis. The mice were infected i.p. with virulent L. monocytogenes (strain DP-L4056), which was kindly provided by Thomas W. Dubensky Jr. from Cerus Corp. Mouse survival was monitored thereafter. In some experiments, liver and spleen were harvested 3 days after infection for histological analysis and measurement of bacterial titer by plating of homogenized organs on CHROMagar Listeria plates (BD Diagnostics).

Northern blot analysis. Total RNA was extracted from liver and spleen using an RNeasy Mini Kit (QIAGEN). Purified RNA (10 $\mu \mathrm{g} / \mathrm{sample}$ ) was separated by electrophoresis on a $1.5 \%$ denaturing agarose gel. The fractionated RNA was then transferred onto a Hybond- $\mathrm{N}^{+}$membrane (Amersham Pharmacia Biotech), and blotted with ${ }^{32} \mathrm{P}-$ labeled full-length mouse LIGHT, HVEM, LT $\beta$ R, or GAPDH cDNA probe using the a Rediprime kit (Amersham Pharmacia Biotech).

ELISA specific to mouse LIGHT. Two distinct LIGHT-specific Abs, ML163 and ML209, were established as previously described (5). For LIGHT-spe- cific ELISA, ML163 (2 $\mu \mathrm{g} / \mathrm{ml})$ was coated on the plate, and biotin-conjugated ML209 $(5 \mu \mathrm{g} / \mathrm{ml})$ was used as detection Ab. ELISA was conducted according to the procedures described previously (5). A linear standard curve was obtained with LIGHT-flag fusion protein as a positive control.

In vitro gene expression and hydrodynamic injection. Lipofectamine 2000 (Invitrogen Corp.) was used for gene transfection into 293T cells according to the manufacturer's instructions. For in vivo gene expression, hydrodynamic injection of plasmids, in which $20 \mu \mathrm{g}$ of plasmid DNA in $2 \mathrm{ml}$ PBS is rapidly injected into the tail vein, was performed as previously described (27). A sublethal dose of ConA $(12.5 \mathrm{mg} / \mathrm{kg})$ was combined in the diluents in some experiments.

Generation of LIGHT mutants. A plasmid encoding a single-amino-acid substitution of Phe for Tyr173 (Y173F) was generated by 2-step PCR in which wild-type mouse LIGHT cDNA was used as the template. First, overlapping oligonucleotide primers encoding the desired mutations were synthesized, and 2 flanking $5^{\prime}$ and $3^{\prime}$ primers were designed with XbaI and BamHI restriction sites, respectively. Appropriate regions of cDNA were initially amplified using corresponding overlapping and flanking primers. Then, using the flanking $5^{\prime}$ and $3^{\prime}$ primers, fragments whose sequences overlapped were fused together and amplified. PCR product was digested with XbaI and BamHI and ligated into XbaI/BamHI-digested pcDNA3.1 vectors (Invitrogen Corp.). Plasmids encoding LIGHTAL mutant were designed to remove amino acids from Leu63 to Asp84 of LIGHT. LIGHTAL was similarly constructed by 2 -step PCR using overlapping oligonucleotide primers encoding the sequences adjacent to the desired deletion. To verify the accuracy of mutation, both mutants were sequenced using an $\mathrm{ABI}$ PRISM 310 Genetic Analyzer (Applied Biosystems).

Statistics. ALT levels and LIGHT levels in the serum were compared between groups using 2-tailed Student's $t$ test. Survival experiments are shown as Kaplan-Meier survival curves and analyzed using the log rank test. $P$ values less than 0.05 were considered statistically significant in both tests.

\section{Acknowledgments}

We thank Jennifer Osborne for editing the manuscript. We would like to acknowledge Masao Ichikawa and Cecilia A. Rietz for technical help. This work was supported in part by grants from the NIH (CA85721 to L. Chen).

Received for publication October 7, 2005, and accepted in revised form January 31, 2006.

Address correspondence to: Lieping Chen or Koji Tamada, 1550 Orleans Street, CRB II, Room 2.11, Baltimore, Maryland 21205, USA. Phone: (410) 502-0957; Fax: (410) 502-0961; E-mail: lchen42@jhmi.edu (L. Chen). Phone: (410) 502-0958; Fax: (410) 502-0961; E-mail: ktamada1@jhmi.edu (K. Tamada).
1. Aggarwal, B.B. 2003. Signalling pathways of the TNF superfamily: a double-edged sword. Nat. Rev. Immunol. 3:745-756.

2. Mauri, D.N., et al. 1998. LIGHT, a new member of the TNF superfamily, and lymphotoxin alpha are ligands for herpesvirus entry mediator. Immunity. 8:21-30.

3. Tamada, K., et al. 2000. LIGHT, a TNF-like molecule, costimulates $\mathrm{T}$ cell proliferation and is required for dendritic cell-mediated allogeneic $\mathrm{T}$ cell response. J. Immunol. 164:4105-4110.

4. Yu, K.Y., et al. 1999. A newly identified member of tumor necrosis factor receptor superfamily (TR6) suppresses LIGHT-mediated apoptosis. J. Biol. Chem. 274:13733-13736.

5. Tamada, K., et al. 2000. Modulation of T-cell-mediated immunity in tumor and graft-versus-host disease models through the LIGHT co-stimulatory pathway. Nat. Med. 6:283-289.

6. Scheu, S., et al. 2002. Targeted disruption of LIGHT causes defects in costimulatory $\mathrm{T}$ cell activation and reveals cooperation with lymphotoxin beta in mesenteric lymph node genesis. J. Exp. Med. 195:1613-1624.

7. Tamada, K., et al. 2002. Cutting edge: selective impairment of CD8+ T cell function in mice lacking the TNF superfamily member LIGHT. J. Immunol. 168:4832-4835.

8. Ye, Q., et al. 2002. Modulation of LIGHT-HVEM costimulation prolongs cardiac allograft survival. J. Exp. Med. 195:795-800.

9. Rooney, I.A., et al. 2000. The lymphotoxin-beta receptor is necessary and sufficient for LIGHTmediated apoptosis of tumor cells. J. Biol. Chem. 275:14307-14315.
10. Zhai, Y., et al. 1998. LIGHT, a novel ligand for lymphotoxin beta receptor and TR2/HVEM induces apoptosis and suppresses in vivo tumor formation via gene transfer. J. Clin. Invest. 102:1142-1151.

11. Lee, W.H., et al. 2001. Tumor necrosis factor receptor superfamily 14 is involved in atherogenesis by inducing proinflammatory cytokines and matrix metalloproteinases. Arterioscler. Thromb. Vasc. Biol. 21:2004-2010.

12. Kim, W.J., et al. 2005. LIGHT is involved in the pathogenesis of rheumatoid arthritis by inducing the expression of pro-inflammatory cytokines and MMP-9 in macrophages. Immunology. 114:272-279.

13. Wang, J., et al. 2004. Dysregulated LIGHT expression on $\mathrm{T}$ cells mediates intestinal inflammation and contributes to IgA nephropathy. J. Clin. Invest. 113:826-835. doi:10.1172/JCI200420096. 
14. Wang, J., et al. 2005. The critical role of LIGHT in promoting intestinal inflammation and Crohn's disease. J. Immunol. 174:8173-8182.

15. Granger, S.W., Butrovich, K.D., Houshmand, P., Edwards, W.R., and Ware, C.F. 2001. Genomic characterization of LIGHT reveals linkage to an immune response locus on chromosome 19p13.3 and distinct isoforms generated by alternate splicing or proteolysis. J. Immunol. 167:5122-5128.

16. Morel, Y., et al. 2000. Reciprocal expression of the TNF family receptor herpes virus entry mediator and its ligand LIGHT on activated T cells: LIGHT down-regulates its own receptor. J. Immunol. 165:4397-4404.

17. Yin, X.M., and Ding, W.X. 2003. Death receptor activation-induced hepatocyte apoptosis and liver injury. Curr. Mol. Med. 3:491-508.

18. Shaikh, R.B., et al. 2001. Constitutive expression of LIGHT on T cells leads to lymphocyte activation, inflammation, and tissue destruction. J. Immunol. 167:6330-6337.

19. Wang, J., et al. 2001. The regulation of $\mathrm{T}$ cell homeostasis and autoimmunity by $\mathrm{T}$ cell-derived LIGHT. J. Clin. Invest. 108:1771-1780. doi:10.1172/ JCI200113827.

20. Lowes, K.N., Croager, E.J., Abraham, L.J., Olynyk, J.K., and Yeoh, G.C. 2003. Upregulation of lymphotoxin beta expression in liver progenitor (oval) cells in chronic hepatitis C. Gut. 52:1327-1332.

21. Chen, C.M., You, L.R., Hwang, L.H., and Lee, Y.H. 1997. Direct interaction of hepatitis C virus core protein with the cellular lymphotoxin-beta receptor modulates the signal pathway of the lymphotoxin-beta receptor. J. Virol. 71:9417-9426.

22. Matsumoto, M., et al. 1997. Hepatitis C virus core protein interacts with the cytoplasmic tail of lymphotoxin-beta receptor. J. Virol. 71:1301-1309.

23. You, L.R., Chen, C.M., and Lee, Y.H. 1999. Hepatitis $\mathrm{C}$ virus core protein enhances NF-kappaB signal pathway triggering by lymphotoxin-beta receptor ligand and tumor necrosis factor alpha. J. Virol. 73:1672-1681.

24. Chen, M.C., Hsu, T.L., Luh, T.Y., and Hsieh, S.L. 2000. Overexpression of bcl-2 enhances LIGHTand interferon-gamma-mediated apoptosis in Hep3BT2 cells. J. Biol. Chem. 275:38794-38801.

25. Anders, R.A., Subudhi, S.K., Wang, J., Pfeffer, K., and Fu, Y.X. 2005. Contribution of the lymphotoxin beta receptor to liver regeneration. J. Immunol. 175:1295-1300.

26. Tiegs, G., Hentschel, J., and Wendel, A. 1992. A T celldependent experimental liver injury in mice inducible by concanavalin A. J. Clin. Invest. 90:196-203.

27. Knapp, J.E., and Liu, D. 2004. Hydrodynamic deliv- ery of DNA. Methods Mol. Biol. 245:245-250.

28. Takeda, K., et al. 2000. Critical contribution of liver natural killer T cells to a murine model of hepatitis. Proc. Natl. Acad. Sci. U. S. A. 97:5498-5503.

29. Toyabe, S., et al. 1997. Requirement of IL-4 and liver NK1 + T cells for concanavalin A-induced hepatic injury in mice. J. Immunol. 159:1537-1542.

30. Seaman, W.E., Sleisenger, M., Eriksson, E., and Koo, G.C. 1987. Depletion of natural killer cells in mice by monoclonal antibody to NK-1.1. Reduction in host defense against malignancy without loss of cellular or humoral immunity. J. Immunol. 138:4539-4544.

31. von der Weid, T., Beebe, A.M., Roopenian, D.C., and Coffman, R.L. 1996. Early production of IL-4 and induction of Th2 responses in the lymph node originate from an MHC class I-independent CD4+NK1.1- T cell population. J. Immunol. 157:4421-4427.

32. Chen, Y.H., Chiu, N.M., Mandal, M., Wang, N., and Wang, C.R. 1997. Impaired NK1+ T cell development and early IL-4 production in CD1-deficient mice. Immunity. 6:459-467.

33. Pamer, E.G. 2004. Immune responses to Listeria monocytogenes. Nat. Rev. Immunol. 4:812-823.

34. Wang, Y., et al. 2005. The role of herpesvirus entry mediator as a negative regulator of T cell-mediated responses. J. Clin. Invest. 115:711-717. doi:10.1172/ JCI200522982.

35. Sedy, J.R., et al. 2005. B and T lymphocyte attenuator regulates $\mathrm{T}$ cell activation through interaction with herpesvirus entry mediator. Nat. Immunol. 6:90-98.

36. Matsui, H., Hikichi, Y., Tsuji, I., Yamada, T., and Shintani, Y. 2002. LIGHT, a member of the tumor necrosis factor ligand superfamily, prevents tumor necrosis factor-alpha-mediated human primary hepatocyte apoptosis, but not Fas-mediated apoptosis. J. Biol. Chem. 277:50054-50061.

37. Browning, J.L., et al. 1997. Characterization of lymphotoxin-alpha beta complexes on the surface of mouse lymphocytes. J. Immunol. 159:3288-3298.

38. Schneider, K., Potter, K.G., and Ware, C.F. 2004 Lymphotoxin and LIGHT signaling pathways and target genes. Immunol. Rev. 202:49-66.

39. Force, W.R, etal. 2000. Discrete signaling regions in the lymphotoxin-beta receptor for tumor necrosis factor receptor-associated factor binding, subcellular localization, and activation of cell death and NF-kappaB pathways. J. Biol. Chem. 275:11121-11129.

40. Chang, Y.H., Chao, Y., Hsieh, S.L., and Lin, W.W. 2004. Mechanism of LIGHT/interferon-gammainduced cell death in HT-29 cells. J. Cell. Biochem. 93:1188-1202.

41. Kuai, J., et al. 2003. Endogenous association of
TRAF2, TRAF3, cIAP1, and Smac with lymphotoxin beta receptor reveals a novel mechanism of apoptosis. J. Biol. Chem. 278:14363-14369.

42. Chen, M.C., et al. 2003. The role of apoptosis signalregulating kinase 1 in lymphotoxin-beta receptormediated cell death. J. Biol. Chem. 278:16073-16081.

43. Kobayashi, N., Kuramoto, T., Yamaoka, K., Hashida, M., and Takakura, Y. 2001. Hepatic uptake and gene expression mechanisms following intravenous administration of plasmid DNA by conventional and hydrodynamics-based procedures. J. Pharmacol. Exp. Ther. 297:853-860.

44. Mohler, K.M., et al. 1994. Protection against a lethal dose of endotoxin by an inhibitor of tumour necrosis factor processing. Nature. 370:218-220.

45. Wielockx, B., et al. 2001. Inhibition of matrix metalloproteinases blocks lethal hepatitis and apoptosis induced by tumor necrosis factor and allows safe antitumor therapy. Nat. Med. 7:1202-1208.

46. Luzina, I.G., et al. 2003. Occurrence of an activated, profibrotic pattern of gene expression in lung CD8+ T cells from scleroderma patients. Arthritis Rheum. 48:2262-2274.

47. Arrunategui-Correa, V., Lenz, L., and Kim, H.S. 2004. CD1d-independent regulation of NKT cell migration and cytokine production upon Listeria monocytogenes infection. Cell Immunol. 232:38-48.

48. Ranson, T., et al. 2005. Invariant V alpha 14+ NKT cells participate in the early response to enteric Listeria monocytogenes infection. J. Immunol. 175:1137-1144.

49. Exley, M.A., and Koziel, M.J. 2004. To be or not to be NKT: natural killer T cells in the liver. Hepatology. 40:1033-1040.

50. Diao, H., et al. 2004. Osteopontin as a mediator of NKT cell function in T cell-mediated liver diseases. Immunity. 21:539-550.

51. Zheng, S.J., Wang, P., Tsabary, G., and Chen, Y.H. 2004. Critical roles of TRAIL in hepatic cell death and hepatic inflammation. J. Clin. Invest. 113:58-64. doi:10.1172/JCI200419255.

52. Desbarats, J., and Newell, M.K. 2000. Fas engagement accelerates liver regeneration after partial hepatectomy. Nat. Med. 6:920-923.

53. Yamada, Y., Kirillova, I., Peschon, J.J., and Fausto, N. 1997. Initiation of liver growth by tumor necrosis factor: deficient liver regeneration in mice lacking type I tumor necrosis factor receptor. Proc. Natl. Acad. Sci. U. S. A. 94:1441-1446.

54. Wilcox, R.A., et al. 2002. Provision of antigen and CD137 signaling breaks immunological ignorance, promoting regression of poorly immunogenic tumors. J. Clin. Invest. 109:651-659. doi:10.1172/ JCI200214184. 